RESEARCH ARTICLE

\title{
Implementation of Preventive Actions to Control Carbapenem-Resistant Enterobacteriaceae and MDR Gram Negatives at a Neurological Hospital
}

\author{
Monica Bricchi ${ }^{1}$, Annalia Asti ${ }^{2}$, Alessia Pascale ${ }^{3}$, Franco Andreini ${ }^{1}$, Milena Arghittu ${ }^{4}$, Dario Caldiroli ${ }^{5}$, Carla \\ Carozzi $^{5}$, Anna Pavan ${ }^{1}$ \\ ${ }^{7}$ Fondazione IRCCS Istituto Neurologico "Carlo Besta", Milan, Italy; \\ ${ }^{2}$ Department of Infectious disease, IRCCS Policlinico San Matteo, University of Pavia, Pavia Italy; \\ ${ }^{3}$ Department of Drug Sciences, Pharmacology Section, University of Pavia, Pavia Italy; \\ ${ }^{4}$ Fondazione IRCCS Ca' Granda - Ospedale Maggiore Policlinico, Milan, Italy. \\ ${ }^{5}$ Fondazione IRCCS Istituto Neurologico 'Carlo Besta', Intensive Care Unit, Milan, Italy
}

\begin{abstract}
Objectives: Objective: The aim of this study is to describe the epidemiological surveillance of Carbapenem-Resistant Enterobacteriaceae (CRE) effected at the Neurological Hospital Carlo Besta, Milano, Italy. The primary causes in our division of multidrug-resistant (MDRs) infections in 2016 were K. pneumoniae and A. baumanniï; the preventive actions have ensured the reduction of their incidence in 2017 and allowed us to discover the microbiological sentinel events (SE) and start appropriate precautionary behavior and isolation.

Methods: The setting is inpatient care with MDR infections and the aim of the procedure is to formalize the management mode, reporting and transmission of $\mathrm{SE}$, collected at the end of study period for previous years from January $1^{\text {st }}, 2015$ to December $31^{\text {st }}, 2017$.

Results: It should be noted that of a total of 5772 hospitalizations detected in 2016 there was an increase in rate of $A$. baumannii compared to the rate found in 2015 for 5679 hospitalizations and in 2017 for 5682 hospitalizations; the same trend was observed for $K$. pneumoniae. For as regards $P$. aeruginosa carbapenemase producing or MDR, the rates were lower in 2016 and in 2017.
\end{abstract}

Conclusions: The main goals that we achieved in the three years considered are the decreased incidence of healthcare-associated infections (HAls) and the decreased incidence of multidrug-resistant (MDR) microorganisms. These actions were of course associated with the control of antibiotic pressure and a careful stewardship. The strict surveillance of HAls is fundamental to control the growing reservoir within hospital communities. J Microbiol Infect Dis 2020; 10(1):1-9.

Keywords: Carbapenem-resistant Enterobacteriaceae, Carbapenem-resistant Acinetobacter baumannii, Sentinel Event, Healthcare Associated Infections, Extended-spectrum B-lactamase, MDR

\section{INTRODUCTION}

The most obvious problems concerning healthcare associated infections (HAls) are inadequate working conditions, the exposure to risk factors such as high-risk medical interventions and invasive devices, and the unsuccessful attempt of the hospital's efforts to prevent infections that require a particular investigation [1]. HAls developed 48 hrs after hospital admission of the patient, and are dependent on two key factors: a) decreased host defenses and colonization by microorganisms, and b) infected or colonized patients who frequently serve as reservoirs of bacterial infections. The increasing use of carbapenems, caused by the widespread diffusion of ESBL (extended-spectrum Blactamase)-producing Enterobacteria, has led to

Correspondence: Dr. Annalia Asti, Department of Infectious disease, IRCCS Policlinico San Matteo, University of Pavia, Pavia Italy 
a rise in resistance in $P$. aeruginosa and $A$. baumannii and in carbapenemase-producing Enterobacteriaceae [2,3]. Currently, the most alarming data are the increasing resistance to carbapenems of $K$. pneumoniae, a more serious problem than ESBLs since there are no effective and manageable antibiotics [4] to treat infections caused by CRE (carbapenem-resistant Enterobacteriaceae) microorganisms [5]. CRE are responsible for many hospital-acquired infections that are difficult to treat, resulting in longer hospital staying, higher costs and high mortality rates; the spread of CRE bacteria is a global phenomenon that sees Italy at the third place [5-7].

At the Neurological hospital Besta, the progressive reduction of incidence rate of alert microorganisms isolated in neurological and neurosurgical patient and generally for microorganisms transferred by contact, were associated with the implementation of preventive actions defined by the CDC [2].

From 2012 the major items have changed such as the inter-facility communication that has been added to interventions; in a previous work we have shown that the number of isolations detected up to December 31st 2014, was significantly reduced and in particular, the rates of patients carrying MDR micro-organism or with specific resistance decreased.

The main goals we achieved in 2014 were: 1) that over $80 \%$ of sentinel events (SE) were found to be from colonization and contamination, only when cultural examinations for SE were shown to be negative did the transfer of patients to other facilities proceed; the actions taken to prevent and their effectiveness on $A$. baumannii and $K$. pneumoniae, which were the primary causes in our division of multidrug-resistant (MDR) infections in 2012, and often associated with co-infection, has ensured a reduction in the incidence of these microorganisms, and let us detect microbiological SEs; 2) the consolidation of an antibiotics policy, characterized by antibiotic selection and the implementation of de-escalation [1].

Namely, for SEs we mean the individual first investigations of microorganisms that are included in hospital surveillance program for their particular antibiotic resistance; the symptoms of the disease may appear or not, while the individual concerned is hospitalized for this reason, we have to trigger appropriate precautionary behavior and isolation. It is important to note that prolonged patient-days necessitate a number of medical procedures that increase the possibility of transferring hospital infectious pathogens [8,9].

Concerning Antimicrobial Stewardship (ABS), it is important to adjust the antibiotic treatment to the individual patient based on the clinical findings (severity of disease, relevant comorbidities), microbiological efficacy, pharmacodynamics and pharmacokinetics [10], with the purpose to reduce selective pressure, resistance to antibiotics and to decrease unnecessary costs. Of essential priority are bacteria resistant to more than three drug groups that represent a particular threat for patients whose assistance requires devices and catheters; notably, the distribution of pathogens among hospitals generally reflect the hospital characteristics [11].

For this reason it is really important to counteract current trends because we could face a return to the pre-antibiotic era, with simple wounds and infections causing significant harm and even death, and routine medical procedures becoming at very high risk [12].

The main aim of this retrospective observational study was to evaluate the incidence of SE from 2015 to 2017 and the policy of retention of the antibiotic therapy.

\section{METHODS}

Setting: this study, approved by the local ethics committee (protocol no. 25E/24/2015), was performed at the Fondazione IRCCS, Istituto Neurologico Carlo Besta, Milano, Italy.

Study design: A cohort study with retrospective data on SEs and determinants, including interventions, were collected at the end of study period for previous years and it was performed to examine the incidence of SE from January 1st, 2015 to December $31^{\text {st }}$, 2017.

Participants: the selection criteria included patients of all ages affected by different neurological and neurosurgical pathologies carrying MDR or with specific resistance; authors followed a check list approved by the Italian hospital infection committee (CIO) of the Foundation on indication of Regione Lombardia (Table 1).

Hospital characteristics (year 2017): The Besta Neurological Institute is a regional and national center of excellence for the treatment of neurological diseases (total number: 213 beds), 
with an average of 5797 both admissions and day hospital/day surgery, over 37031 specialist outpatient visit and 2421 neurosurgery procedures. The foundation treats oncological, degenerative and rare neurological diseases affecting both children and adults. It is highly active in the field of neurosurgery and carries out clinical and basic translational research to improve treatment and diagnosis.

Admission was accepted as the point at which a person begins an episode of care, by arriving at an inpatient care. Informed consent was obtained from all patients enrolled in the study.

For infection control, measures and procedures regarding the incidence of SEs; microbiological and epidemiological information on SEs, analysis of the factors that are determinants for the occurrence of the same events, individuation of SEs and implementation of surveillance measures, checking of the implementation of preventive actions and their effectiveness, notification of SEs to departments when microorganisms are isolated, use of checklist protocol for HAls prevention, implementation of procedures for specific microorganisms and evaluation of the actions taken to reduce risk factors defined in CDC 2012/2015 were considered (CRE Toolkit Guidance for control of CRE) [2].

During routine visit, microbiological samples were collected from patients with clinical symptoms: respiratory tract secretions, urine, blood culture, central venous catheter (CVC), cerebrospinal fluid, stool, swab from surgical wounds, cervical and urethral specimens were sent to laboratories for clinical purposes. Data were obtained from microbiological isolation in culture tests and identification with Maldi-TOF [13] and supplementary tests. Antimicrobial susceptibility test towards each antimicrobial agent is reported according to international values. Since 2012, the adoption has begun of a new European breakpoint proposed by the EUCAST guidelines [14].

The outcome definition are as follows: 1) routine microbial sampling, in terms of periodicity, was carried out for the intensive care unit (ICU), where surveillance of SEs was performed twice a week, every week for up to three successive negative outcomes, 2) and for all other units was performed once a week; to underline that antibiotic administration exerts selection pressure, promoting the development of resistant microorganisms and modifying the bacterial sensitivity profile.

Antibiotic-free days: number of days without therapy starting from the day of the last administration. They are defined as the number of days, within 28 days after blood culture collection, on which the patient did not receive any antibiotic treatments; any patient dying within 28 days of blood culture collection was assigned zero antibiotic-free days.

De-escalation therapy: the narrowest spectrum as possible, according to available microbiological results; the choice of empirical antibiotic therapy should be performed according to local guidelines.

Data Analysis: Categorical variables were described by rates or percentages, and expressed as mean + SEM of data collected between 2015 and 2017.

For statistical analysis, the GraphPad Instat Statistical Package (version 3.05 GraphPad software, San Diego, CA) was used. The data were analyzed by analysis of variance (ANOVA); followed, when significant, by an appropriate post hoc comparison test as indicated in the legends. Differences were considered statistically significant when $p$ values $<0.05$.

\section{RESULTS}

It should be noted that of a total of 5772 hospitalizations detected up to 31 December 2016 there was an increase in rate of $A$. baumannii compared to the rate found in 2015 for 5679 hospitalizations and in 2017 for 5682 hospitalizations. The same trend was observed for $K$. pneumoniae; for as regards $P$. aeruginosa carbapenemase producing or MDR, the rates were lower in 2016 and in 2017 (Table 1).

Table 2 reports the specific rates relative to each microbiological SE found from the year 2015 to 2017 at the Carlo Besta Neurological Hospital.

The 2016 SE rate is related to a percentage of antibiotic free days in 33\% long-term ICU patients staying more than 72 hours. The following year, 2017, the SE rate decreased returning similar to that of 2015 and is related to 
a percentage of antibiotic free days of over $40 \%$ (Table 1).

Indeed, SEs rate and HAls rate are significantly more elevated in ICU when compared to the respective SEs and HAls detected in the other hospital units. Statistical data are in agreement with the clinical evidences: when the HAls decrease fewer SEs are detectable (Fig. 1 A). In particular, concerning SEs, it should be emphasized that there are no significant differences between SE-CRE and MDR when considering the single microorganism; on the contrary, the significance is relevant when comparing the total SEs number with each single microorganism (Figure 1B). Specifically, A. baumannii, which was present almost in two occasions, originated little but significant clusters since it is known that this organism is more often involved in colonization rather than in infection; however, it should be underscored that the statistic relevance is always in contrast with the clinical relevance. For the three years considered (2015-17), the incidence of SEs CRE and MDR is not statistically significant (Fig. 2B). In the period 2015-2017 the prevalence of HAls is due to urinary tract infections (UTI) in all hospital units (Fig. 2A); this kind of infection is also prevalent in patients hospitalized in ICU. Less frequently HAls were associated with pneumonia, sepsis (LCBI), surgical site infections (SSI), or intracranial brain abscess (IC-MEN) [14]. In agreement with literature data, intensive care is the environment where there is the highest incidence of SEs on 1.000 hospitalizations, this considering that SEs and HAls are both present in the ICU and in the other hospital units (Table 3 ). In addition, the trend of the SEs rates in the ICU, for the three years considered, follows the trend of the total SEs.

\section{DISCUSSION}

The implementation of preventive actions to reduce antibiotic-resistance in MDR and CRE microorganisms allowed us to reduce the Gram $-/+$ alert, and also to control HAls both of alert/non alert microorganisms and generally for microorganisms transferred by contact, associated with guidance for control of CRE [14]. These actions were of course associated with the control of antibiotic pressure and a careful stewardship.
In Figure 3 is shown the trend of a difference between SEs and HAls regarding methicillinresistant $S$. aureus (MRSA). The data are referred to all the hospital wards and the ICU; the low numbers indicate that the antibioticresistance phenomenon in this Gram + microorganism is very limited and secondary. The strict control carried out of measures to prevent or contain $A$. baumannii, (Table 1), which was the most important cause found in our department of HAls MDR in 2012, have assured a reduction in the incidence of the same rate of patients carrying MDRs or KPC microorganism (Table 3).

Table 1. SE KPC or MDR rate and ICU antibiotic Free days rate.

\begin{tabular}{|c|c|c|c|}
\hline Sentinel Events & 2015 & 2016 & 2017 \\
\hline A. baumannii MDR & $2 / 5679$ & $13 / 5772$ & $9 / 5682$ \\
\hline $\begin{array}{l}\text { K. pneumoniae KPC } \\
\text { or MDR }\end{array}$ & $8 / 5679$ & $16 / 5772$ & $10 / 5682$ \\
\hline $\begin{array}{l}\text { P. aeruginosa KPC } \\
\text { or MDR }\end{array}$ & $10 / 5679$ & 9/5772 & $4 / 5682$ \\
\hline Tot SE KPC or MDR & 3.45 & 6.55 & 4.04 \\
\hline $\begin{array}{l}\text { Antibiotic free days } \\
(\%)\end{array}$ & nv & 33 & 44 \\
\hline SE ICU/SE Total (\%) & $26 / 70$ & $40 / 82$ & $22 / 52$ \\
\hline
\end{tabular}

In 2015 the total incidence of HAls is similar to that recorded in 2014 (not shown) but, probably in relation to a more serious case mix, the trend increased in 2016. From the second semester of 2016, concomitantly with a selective antibiotic stewardship in ICU, based on a rising to antibiotic-free days in long-term ICU patients, we assisted to a concomitant reduction in HAls and isolated alert microorganisms. The trend of HAls and alert microorganisms are the same for the considered three years. As it can be seen from the above epidemiological analysis, the microbiological SEs that have burdened the most are those present in ICU and in the wards.

These are for the most part post-surgical patients with both general and neurological conditions compromised, carriers of CVC, urinary catheter, and drainages. They have been/are subjected, contextually with the SE isolation, to prolonged antibiotic therapies. 
A

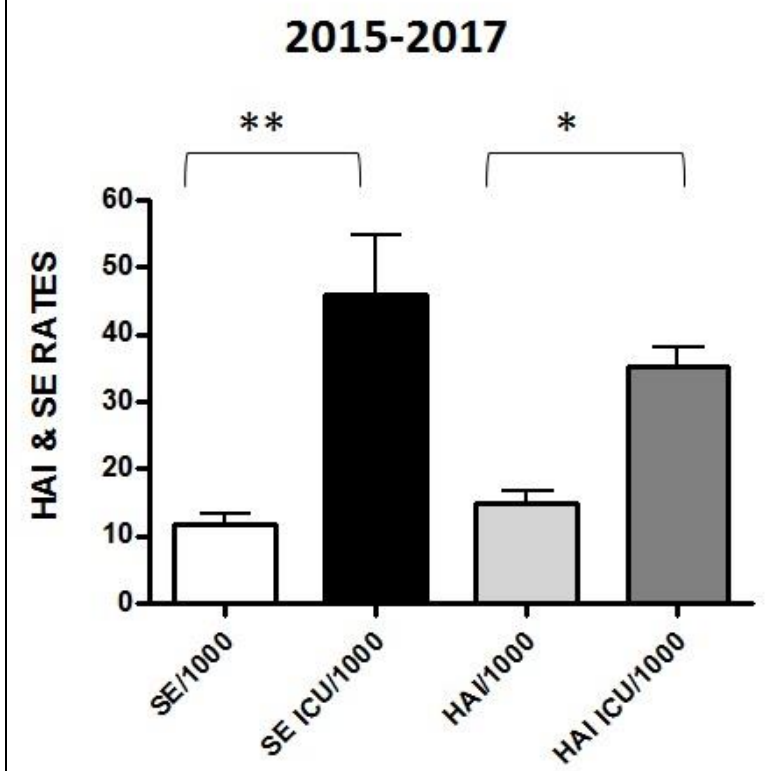

B

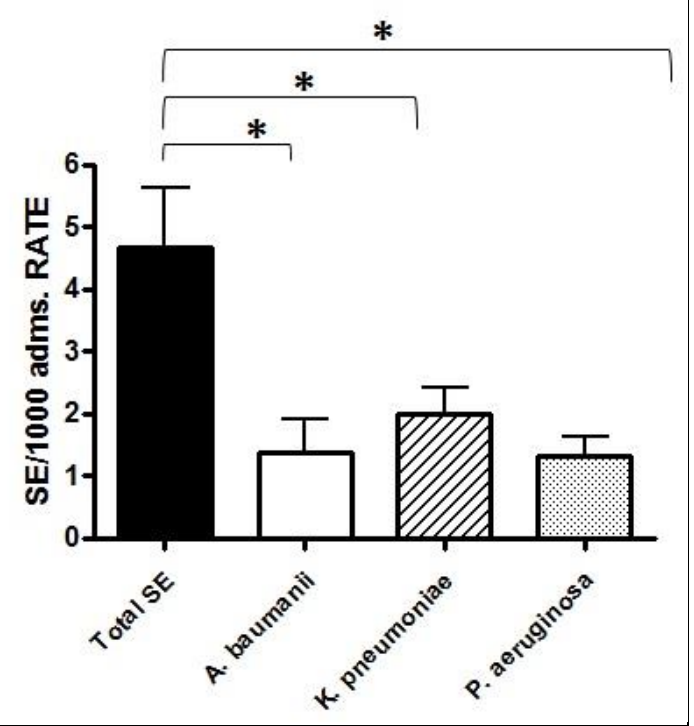

Figure 1. A) SEs and HAls rates. ${ }^{*} p<0.05,{ }^{* *} p<0.01$; Newman-Keuls multiple comparison test, $n=3$.

B) No significant differences among SE-CRE and MDRs. ${ }^{*} p<0.05$, Newman-Keuls Multiple Comparison test , $n=3$.

\section{A}
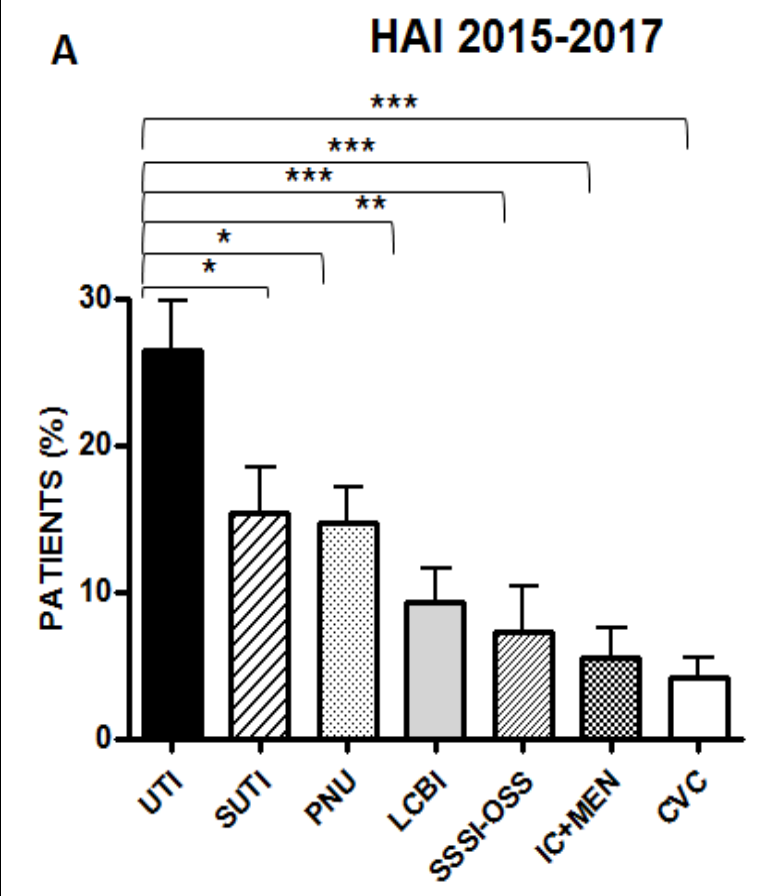

B

SE-KPC \& MDR

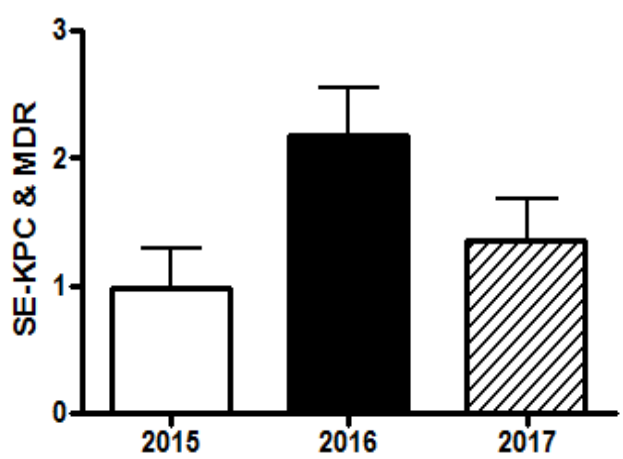

Figure 2. A) Prevalence of HAls. ${ }^{*} p<0.05,{ }^{* *} p<0.01 ;{ }^{* *} p<0.001 ;{ }^{* * *}$, Newman-Keuls multiple comparison test.

B) SE CRE \& MDRs incidence. 
Table 2. SE involved at Neurological Hospital.

\begin{tabular}{|c|c|c|c|c|c|c|}
\hline Microrganismo & SE & $\begin{array}{l}\text { Rate* } 2015_{(n=5679)}\end{array}$ & SE & $\begin{array}{l}\text { Rate }^{*} 2016 \\
(n=5772)\end{array}$ & SE & $\begin{array}{l}\text { Rate }^{*} 2017, \\
(n=5682)\end{array}$ \\
\hline Acinetobacter baumannii non MDR & 2 & 0.3521 & 0 & 0 & 0 & 0 \\
\hline Acinetobacter baumannii MDR & 2 & 0.3521 & 13 & 2.2522 & 9 & 1.5839 \\
\hline Acinetobacter junii Ertapenem- $R$ & & 0 & & 0 & & 0 \\
\hline Burkholderia capacia & & 0 & & 0 & & 0 \\
\hline Candida albicans & & 0 & & 0 & & 0 \\
\hline Corynbacterium species & & 0 & & 0 & & 0 \\
\hline Corynbacterium striatum & & 0 & & 0 & & 0 \\
\hline Enterobacter cloacae & & 0 & & 0 & & 0 \\
\hline Enterococcus VRE & & 0 & & 0 & 1 & 0.1759 \\
\hline Escherichia coli ESBL + & 18 & 3.1695 & 12 & 2.0790 & 13 & 2.2879 \\
\hline Klebsiella pneumoniae MDR & & 0 & & 0 & & 0 \\
\hline Klebsiella pneumoniae KPC & 8 & 1.4086 & 16 & 2.7720 & 10 & 1.7599 \\
\hline Klebsiella pneumoniae ESBL + & 2 & 0.3521 & 10 & 1.7325 & & 0 \\
\hline Klebsiella oxitoca ESBL + & 1 & 0.1760 & & 0 & & 0 \\
\hline Proteus mirabilis ESBL + & & 0 & & 0 & & 0 \\
\hline Providencia stuartii & & 0 & & 0 & & 0 \\
\hline $\begin{array}{l}\text { Pseudomonas aeuroginosa KPC or } \\
\text { MDR }\end{array}$ & 10 & 1.7608 & 9 & 1.5592 & 4 & 0.7039 \\
\hline Salmonella group C1 & & 0 & & 0 & 2 & 0.3519 \\
\hline Serratia marcescens & 17 & 2.9934 & 6 & 1.0395 & 3 & 0.5279 \\
\hline $\begin{array}{l}\text { Staphylococcus aureus OXA R or } \\
\text { MRSA }\end{array}$ & 8 & 1.4086 & 6 & 1.0395 & 6 & 1.0559 \\
\hline Staphylococcus capitis & & 0 & & 0 & & 0 \\
\hline Staphylococcus epidermidis & & 0 & & 0 & & 0 \\
\hline Stapylococcus haemolyticus & & 0 & & 0 & & 0 \\
\hline Staphylococcus hominis & & 0 & & 0 & & 0 \\
\hline Stapylococcus sciurii & & 0 & & 0 & & 0 \\
\hline Stapylococcus warneri & & 0 & & 0 & & 0 \\
\hline Stenotrophomonas maltophilia & 2 & 0.3521 & 6 & 1.0395 & 2 & 0.3519 \\
\hline Aspergillus fumigatus & & 0 & 3 & 0.5197 & 1 & 0.1759 \\
\hline Clostridium difficile & & 0 & & 0 & 1 & 0.1759 \\
\hline Total & 70 & 12.3253 & 81 & 14.0331 & 52 & 9,1509 \\
\hline
\end{tabular}

${ }^{\star}$ Rate for 1000 admissions

Table 3. A) Trend of patients with ICA and SEs present both in the ICU and in hospital units in 2015-2017 B) Rate of patients carrying multi-drug- resistant microorganisms or microorganisms with specific resistance.

\begin{tabular}{lccc}
\hline Variables & 2015 & 2016 & 2017 \\
\hline Patients with ICA & 87 & 103 & 66 \\
Patients with ICA/1000 admission & 15,3 & 17,8 & 11,6 \\
Patients with SE & 52 & 71 & 43 \\
SE/1000 admissions & 12,3 & 71 & 9,2 \\
SE/10000 LOS & 17,5 & 20,6 & 13,2 \\
\hline HAl=Hospital-acquired Infection & SE= sentinel event & LOS= length of stay
\end{tabular}

$\mathrm{HAI}=$ Hospital-acquired Infection $\quad \mathrm{SE}=$ sentinel event $\quad \mathrm{LOS}=$ length of stay

Often, in the ICU patients, the microbiological $\mathrm{SE}$ is present but it is not responsible for the infections affecting the patient itself. As observed by other authors [15], the number of
HAls was higher in male than in female patients, although the reason is not clear $[16,17]$, the higher frequency of sepsis due to Klebsiella species has been associated with a genetic predisposition [18]. 
A higher frequency of CRE could be related to the higher vulnerability of the ICU patients and to their longer staying compared to patients admitted to other units with less intensive level of care [15]. In surgical patients undergoing treatment with invasive surgical devices, proper use of preoperative antibiotic prophylaxis obviates the extension of antibiotic therapy and the implementation of measures required to contain the infection (Table 1).

The infection control interventions that mainly focus on hospitals are less likely to be effective because, for example, microorganism such as Escherichia coli spreads in the community more quickly than Klebsiella pneumoniae [15].

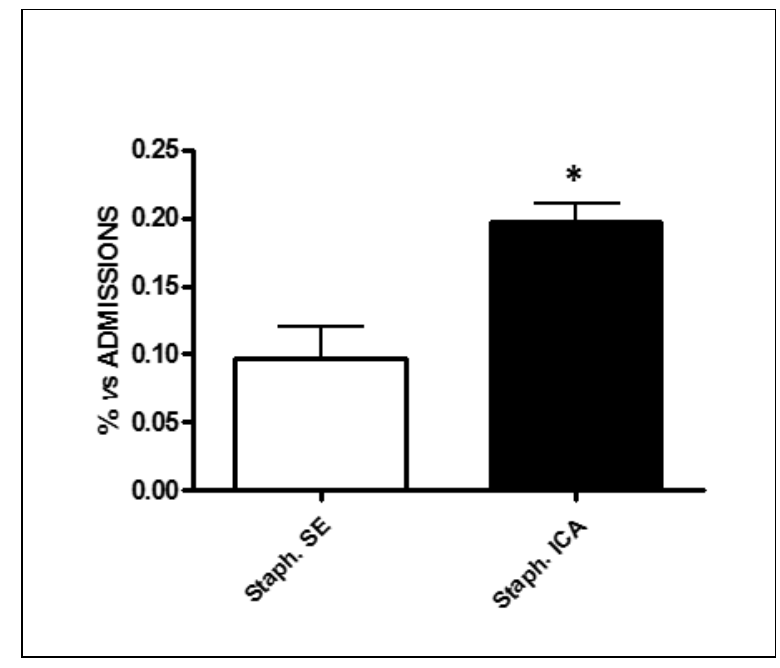

Figure 3. SEs and HAls of the Methicillin-resistant Staphylococcus aureus. The data are concerning all hospital units and the ICU, ${ }^{*} p<0.05$.

The increasing use of carbapenems, caused by the widespread diffusion of ESBL-producing enterobacteria, has caused an increase in resistant Gram-negative bacteria, such as $P$. aeruginosa and $A$. baumannii, and in carbapenemase-producing Enterobacteriaceae [19], but a strict selective antibiotic stewardship has allowed us to increase the number of antibiotic-free days in ICU (Table 1).

Moreover CRE are resistant to virtually all betalactam antibiotics and may contain mechanisms of resistance against a second-line antibiotics such as aminoglycosides and fluoroquinolones.

Regarding UTI infections, older people, also recovered at the neurological unit, with urinary tract infections may have different symptoms than young or middle-aged adults, especially subjects with dementia may not be able to describe symptoms, and fever may not even occur. Sometimes, the only thing that is noticed in patients with UTI is a change in mental status, known as delirium, which is caused by a potentially reversible condition such as infection, hypoglycemia, or drug side effects. Moreover, it has been demonstrated an association between age and the poor response of the immune system [20] and the presence of co-morbidities.

The main study limitation is related to data collection; to the inability to identify all the SEs these are present at the admission of the patient to the hospital since in our hospital there is no emergency room. The SE rate present at the admission of patients sometimes is unknown because is difficult and expensive to submit all patients to a microbiological triage. Indeed, only if patients are considered at risk of HAls or are coming from other hospitals, they are submitted to microbiological investigations, such as biological culture sampling; for this reason, patients that are considered at risk or carrier of SEs are easily discernable. CRE infections are yet associated with poor outcomes and high mortality [21].

\section{Conclusion}

Therefore, more research is needed to fight antimicrobial resistance $[22,23]$, to develop new therapeutic drugs and innovative treatments and products for humans and animals. Most of the microorganisms isolated were either acquired at the hospital, and often associated with intensive care treatment, or sometimes isolated from patients with a previous hospital admission [24, 25]; further, there also seems to be an association with preceding travels outside the country of residence [26]. In this study, there was a consolidation of a rigid antibiotics policy, characterized by a non-treatment of colonization and the implementation of de-escalation [27-29].

Surveillance of healthcare-associated infections allows understanding the sources that are involved to the growing reservoir within hospital communities and, as suggested by other authors, there is a compelling need for urgent interventions by the health authorities [15, 22, 30].

\section{ACKNOWLEDGMENTS}


The authors wish to thank Mrs. Lara Firmo of Infectious Disease, Policlinico San Matteo, Pavia, Italy and Mrs. Rosalind Hendricks of Istituto Besta, Milano, Italy

Declaration of Conflicting Interests: The authors declare that they have no conflict of interest.

Financial Disclosure: No financial support was received.

\section{REFERENCES}

1. Asti A, Marmondi E, Tinelli C, et al. Microbiological sentinel events at a neurological hospital: a retrospective cohort study. J Med Microbiol 2016; 65, 1512-1520.

2. Guidance for control of Carbapenem-resistant Enterobacteriaceae (CRE) Facility Guidance for Control of Carbapenem-resistant Enterobacteriaceae (CRE). November 2015 Update - CRE Toolkit. National Center for Emerging and Zoonotic Infectious Diseases; Division of Healthcare Quality Promotion CDC.

3. Zhang $Y$, Wang $Q$, Yin $Y$, et al. Epidemiology of Carbapenem-Resistant Enterobacteriaceae Infections: Report from the China CRE Network. Antimicrob Agents Chemother 2018; 62 e01882-1817.

4. Meletis G. Carbapenem resistance: overview of the problem and future perspectives. Ther Adv Infect Dis 2016; 3(1), 1521.

5. van Duin D, Doi Y. The global epidemiology of carbapenemase producing Enterobacteriaceae. Virulence 2017; 8(4), 460-469.

6. Global priority list of antibiotic-resistant bacteria to guide research, discovery, and development of new antibiotics.http://www.who.int/medicines/publications/g lobalpriority-list-antibiotic-resistant-bacteria/en/

7. Papp-Wallance KM, Endimiani A, Taracila MA, et al. Carbapenems: past, present and future. Antimicrob Agent Chemother 2011; 4943-4960

8. Hauck, K. \& Zhao, X. How dangerous is a day in hospital? A model of adverse events and length of stay for medical inpatients. Med Care 2011; 49, 10681075.

9. World Health Organization Prevention of HospitalAcquired Infections. a Practical Guide, $2^{\text {nd }}$ edn. Geneva: 2002 WHO/CDS/CSR/EPH.

10. Fritzenwanker M, Imirzalioglu C, Herold S, et al. Treatment Options for Carbapenem- Resistant GramNegative Infections. Dtsch Arztebl Int 2018; 115, 34552.

11. Puzniak L, DePestel DD, Srinivasan A, et al. A Combination Antibiogram Evaluation for Pseudomonas aeruginosa in Respiratory and Blood Sources from ICU and Non-ICU Settings in US.
Hospitals. Antimicrob. Agents Chemother 2019; 63, 4 e02564-18.

12. A European One Health Action Plan against Antimicrobial Resistance (AMR). 2017 European Commission). https://eur-lex.europa.eu/legalcontent/IT/TXT/PDF/?uri=CELEX:52017DC0339\&fro $\mathrm{m}=\mathrm{EN}$

13. Nagy $E$, Becker $S$, Kostrzewa $M$, et al.. The value of MALDI-TOF MS for the identification of clinically relevant anaerobic bacteria in routine laboratories. J Med Microbiol 2012; 61, 1393-1400.

14. European Committee on Antimicrobial Susceptibility Testing (EUCAST). EUCAST guidelines for detection of resistance mechanisms and specific resistances of clinical and/or epidemiological importance, December 2013. http://www.eucast.org/fi leadmin/src/media/PDFs/EUCAST files/

Resistance_mechanisms/EUCAST_detection_of_resi stance_mechanisms_v1.0_20131211.pdf

15. lacchini S, Sabbatucci M, Gagliotti C, et al. A. Bloodstream infections due to carbapenemaseproducing Enterobacteriaceae in Italy: results from nationwide surveillance, 2014 to 2017. Eurosurveill $2019 ; 24,5$.

16. Uslan DZ, Crane SJ, Steckelberg JM, et al. Ageand sex-associated trends in bloodstream infection: a population-based study in Olmsted County, Minnesota. Arch Intern Med 2007; 167(8), 834-839.

17. Kousouli E, Zarkotou O, Politi L, et al. Infection control interventions affected by resource shortages: impact on the incidence of bacteremias caused by carbapenem-resistant pathogens. Eur J Clin Microbiol Infect Dis 2018; 37(1), 43-50.

18. Hubacek JA, Stüber F, Fröhlich D, et al. Gene variants of the bactericidal/permeability increasing protein and lipopolysaccharide binding protein in sepsis patients: gender-specific genetic predisposition to sepsis. Crit Care Med 2001; 29(3), 557-561.

19. Albiger B,. Glasner C, MJ Struelens MJ, et al. The European Survey of Carbapenemase-Producing Enterobacteriaceae (EuSCAPE) working group. Carbapenemase-producing Enterobacteriaceae in Europe: assessment by national experts from 38 countries, May 2015. Euro Surveill 2015; 20(45).

20. Weng NP. Aging of the immune system: how much can the adaptive immune system adapt? Immunity 2006; 24(5),495-499.

21. Codjoe FS and Donkor ES. Carbapenem Resistance: A Review. Med Sci 2018; 6, 1

22. Ministero della Salute (Italian Ministry of Health). Piano Nazionale di Contrasto dell'AntimicrobicoResistenza (PNCAR) 2017-2020. [National Action Plan on Antimicrobial Resistance 2017-2020] Rome: Ministry of Health; 24 Oct 2017. Italian. http://www.salute.gov.it/imgs/C_17_http://www.salute. gov.it/imgs/C. 
23. Goldman DA, Weinstein RA, Wenzel RP, et al. Strategies to Prevent and Control the Emergence and Spread of Antimicrobial-Resistant Microorganisms in Hospitals. A Challenge to Hospital Leadership. JAMA 1996; 275, 234-240

24. Grundmann $\mathrm{H}$, Glasner $\mathrm{C}$, Albiger $\mathrm{B}$, et al.and the European Survey of Carbapenemase-Producing Enterobacteriaceae (EuSCAPE) Working Group. Occurrence of carbapenemase-producing Klebsiella pneumoniae and Escherichia coli in the European survey of carbapenemase-producing Enterobacteriaceae (EuSCAPE): a prospective, multinational study. Lancet Infect Dis 2017; 17, 153163.

25. Mandell GL, Bennett JE, Dolin R, Douglas M. Bennett's Principles and Practice of Infectious Diseases, $7^{\text {th }}$ edn. Oxford, 2010, Elsevier/Churchill Livingstone, United Kingdom.

26. Christoff J, Tolentino J, Mawdsley E, et al. Optimizing empirical antimicrobial therapy for infection due to Gram-negative pathogens in the intensive care unit: utility of a combination antibiogram. Infect Control Hosp Epidemiol 2010; 31, 256-261.

27. Davey $P$, Marwick CA, Scott $C L$ et al. Interventions to improve antibiotic prescribing practices for hospital inpatients Cochrane Database of Systematic Reviews 2017; 9,2.

28. Hojgard S. Antibiotic resistance-why is the problem so difficult to solve? Infect Ecol Epidemiol 2012; 2,18165

29. Abulhasan YB, Rachel SP, Chatillon-Angle MO, et al. Healthcare associated infections in the neurological intensive care-unit: Results of 6 years surveillance study at a major tertiary care center. Am J Infect Control 2018; 46, 656-662. 